\title{
Non-equally-spaced pulse transmission for non-aliasing ultrasonic pulsed Doppler measurement
}

\author{
Hisashi Nishiyama and Kageyoshi Katakura \\ Central Research Laboratory, Hitachi, Ltd., \\ 1-280, Higashi-Koigakubo, Kokubunji, 185 Japan
}

(Received 14 September 1991)

\begin{abstract}
This paper presents a new method of measuring blood velocities and the Doppler frequency distribution that is not limited by the Nyquist frequency. A non-equally-spaced pulse transmission (a staggered pulse repetition time) method is used to calculate the second-order phase difference (velocity) between successive echoes from the blood flow, and the noise-induced fluctuation in the phase difference is suppressed by averaging the phase difference vectors. With conventional pulsed-Doppler equipment, Nyquist's sampling theorem and the relationship between frequency and distance limit the maximum measurable velocity to the reciprocal of twice the pulse repetition time (sampling time). We show theoretically that the proposed method has a larger maximum velocity. When the non-equally-spaced pulse repetition intervals are $T$ and $T+T_{\mathrm{s}}$, the maximum measurable velocity can be increased to $P$ times the conventional value by selecting $T_{\mathrm{s}}$ equal to $T / P(P>1)$. The results of computer simulation with a high signal-to-noise ratio are in good agreement with the results measured using the proposed method. Invivo high-speed blood flow (at over the Nyquist-frequency) in the carotid artery and heart is unambiguously measured without aliasing. To illustrate the feasibility of the proposed method, we also compare the spectrum with that obtained using conventional pulsed-Doppler equipment.
\end{abstract}

Keywords: Pulsed Doppler, Aliasing, CFM, staggered PRT

PACS number: 43. 80. Vj, 48. 80. Qf, 43. 85. -e

\section{INTRODUCTION}

Conventional ultrasound pulsed Doppler equipment using an estimator of the power-weighted mean frequency ${ }^{1)}$ (pulse pair processing) can display real-time two-dimensional images of the blood flow in a human heart. ${ }^{2,3}$ ) This is very useful for immediate diagnosis of cardiac problems. For example, it can indicate the velocity of blood flowing towards the transducer in red and that of blood flowing away from the transducer in blue. However, due to the Nyquist sampling theorem and a limitation created by the relationship between the pulse repetition frequency and the distance, conventional pulsed Doppler equipment has a maximum measurable velocity, within a depth of $15 \mathrm{~cm}$ from the transducer surface, of less than about $1 \mathrm{~m} / \mathrm{s}$. If the velocity of blood flowing towards the transducer over this limit, the velocity cannot be measured correctly but is shown by blue instead of red. Various techniques have been suggested and investigated in order to remove the aliasing problem. ${ }^{4-11)}$

In pulsed-Doppler frequency analysis, aliasing has been removed by reconstructing the true spectrum shape through spectrum component reordering.9.10) For color flow mapping (or 2-D Color Doppler), one method is to use a non-Doppler technique ${ }^{7,8)}$ that takes a time-domain approach to pulsed-Doppler signals. The time cross-correlation technique does not have the above limitation on measuring blood velocity. However, it does have one fault in that peaks may be detected erroneously during large accelerations or under a low-signal-to-noise ratio. Another technique named the aliasing extension 
method $^{11)}$ needs to assume the mean velocity continuity. This technique, however, has a serious fault in that the velocity is not always continuous in the spatial domain under turbulent flow or under a low signal-to-noise ratio in the real clinical situation.

We developed a new method ${ }^{12-15)}$ of measuring velocities over the Nyquist frequency, in which a non-equally-spaced pulse transmission (a staggered pulse repetition time) method is used to calculate the second-order phase difference (velocity) between successive echoes from the blood flow. This method also gives the Doppler frequency distribution by estimating the original de-aliased phase change from the second-order phase difference vectors.

In the area of the severe weather observation, Doppler weather radars had suffered from a similar aliasing problem when measuring a local wind velocity over the Nyquist frequency. A similar method was suggested in this area ${ }^{16)}$ without the capability of frequency distribution analysis.

In the present paper, we apply this method to real clinical situations and discusses its feasibility and effectiveness in the field of the medical ultrasound.

\section{PRINCIPLE}

\subsection{Conventional Method}

Figure 1 shows the conventional pulsed Doppler blood flow measurement system. Ultrasonic pulses are transmitted at an equal time interval $T$ to the blood flow in the heart by an ultrasonic transducer. The same transducer receives the pulses reflected from the blood flow. After phase detection and MTI (Moving Target Indication) filter processing, the velocity is estimated from the output signals
$V_{\mathrm{rn}}$ and $V_{\mathrm{ri}}$ of the phase comparator, which compares the reflected wave with reference signals $\alpha$ ( $\left.=A \sin \omega_{0} t\right)$ and $\alpha^{\prime}\left(=A \cos \omega_{0} t\right)$, as follows:

$$
\begin{aligned}
& V_{\mathrm{rn}}=A_{n} \cos \theta_{n} \\
& V_{\mathrm{ri}}=A_{n} \sin \theta_{n} .
\end{aligned}
$$

Their vector representation is as follows.

$$
V_{n}=V_{\mathrm{rn}}+j V_{\mathrm{ri}}=A_{n} \exp \left(j \theta_{n}\right)
$$

To obtain the phase difference (blood velocity) $\Delta \theta$, the complex multiplication of $V_{n+1}$ and the complex conjugate of $V_{n}$ is carried out, that is,

$$
\begin{aligned}
Y_{n} & =V_{n+1} \cdot \operatorname{conjg}\left[V_{n}\right] \\
& =Y_{n \mathrm{r}}+j Y_{n \mathrm{i}},
\end{aligned}
$$

(where $Y_{n \mathrm{r}}$ : real part, $Y_{n \mathrm{i}}$ : imaginary part).

We thus get the phase difference vector $Y_{n}$. From Eq. (2), we can obtain the phase difference $\Delta \theta$ $\left(=\omega_{\mathrm{d}} T=\arg \left[Y_{n}\right]=\operatorname{Arctan}\left(Y_{n \mathrm{i}} / Y_{n \mathrm{r}}\right)\right.$, where $\omega_{\mathrm{d}}$ is the Doppler angular frequency $(\mathrm{rad} / \mathrm{s}))$. Figure 2 shows the aliasing problem in conventional pulsed Doppler measurement. Pulses are transmitted at equallyspaced $T$ (a fixed PRT). When blood flows towards the transducer at a velocity $V$, there is a phase difference $\Delta \theta$ between two reflected waves. This can be expressed by the relationship $\Delta \theta=2 k V T$, where $k=2 \pi / \lambda$ ( $\lambda$ is wavelength). Therefore, $\Delta \theta$ is related to the blood velocity $V$. The maximum measurable phase difference (velocity) is $\pm \pi$.

Unfortunately, if the blood velocity is very high, there is a big problem because $\Delta \theta$ becomes negative (Fig. 2c). This is why we cannot measure beyond the Nyquist frequency and also explains the opposite blue color using the conventional method. Thus, the maximum measurable Doppler frequency (velocity) of $1 / 2 T$ in the conventional method is reached

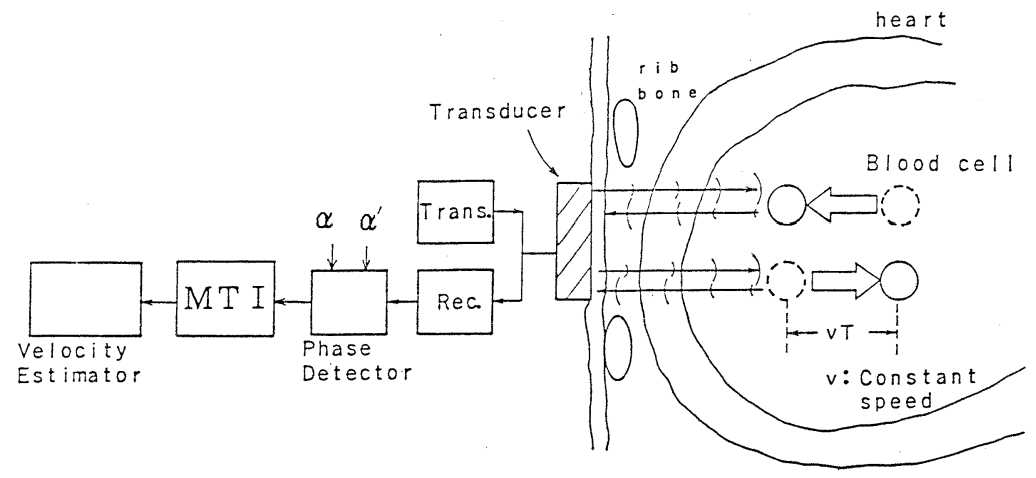

Fig. 1 Pulsed Doppler measurement system. 


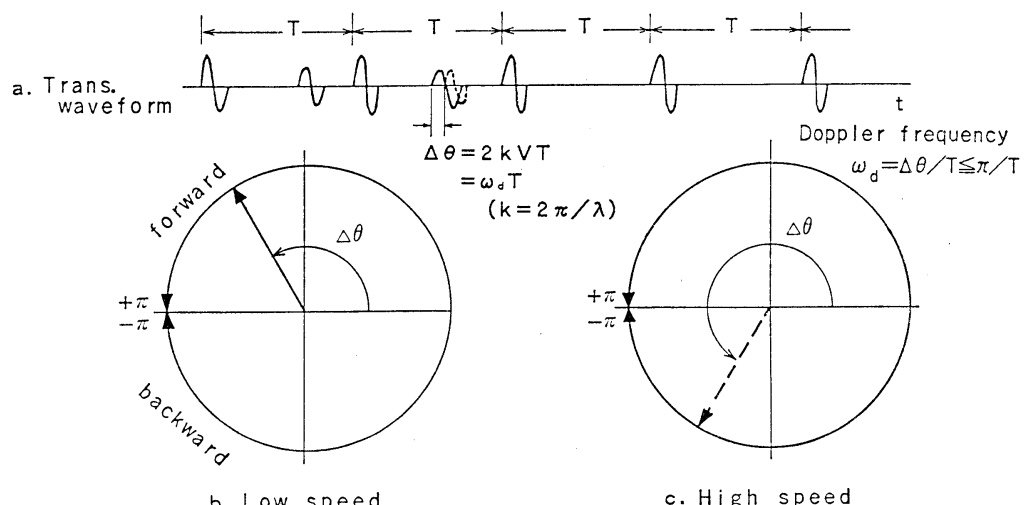

Fig. 2 Alising problem in conventional pulsed Doppler measurement.
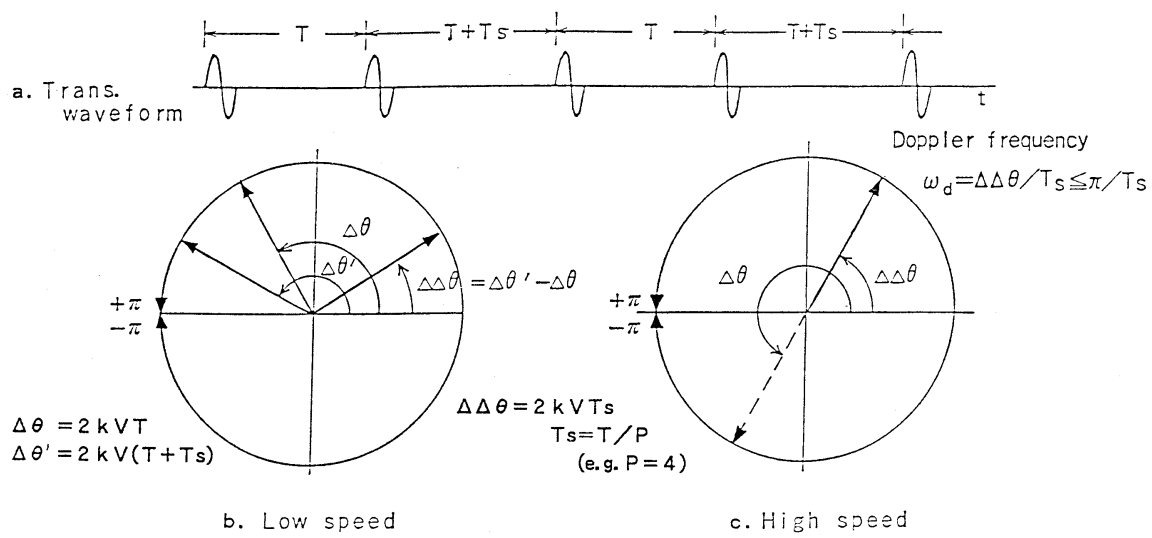

Fig. 3 Non-equally spaced pulse transmission.

when $\Delta \theta$ is $\pi$. If we select a smaller $T$, the maximum measurable velocity will be increased but the measurable depth will be decreased due to overlaid reflected waves. We thus encounter a limitation created by the relationship between the frequency and the depth. The maximum measurable velocity within a depth of $15 \mathrm{~cm}$ from the transducer surface is less than about $1 \mathrm{~m} / \mathrm{s}$. The conventional method cannot unambiguously measure the blood velocities above this limit.

2.2 Non-equally-spaced Pulse Transmission ${ }^{12,13)}$ (a staggered PRT) Method

In the proposed method (Fig. 3), the transmission pulses are non-equally-spaced (Fig. 3a). The phase difference $\Delta \theta$ is for pulse transmission period $T$; the phase difference $\Delta \theta^{\prime}$ is for pulse transmission period $T+T_{\mathrm{s}}$, that is,

$$
\begin{aligned}
& \Delta \theta=\omega_{\mathrm{d}} T \\
& \Delta \theta^{\prime}=\omega_{\mathrm{d}}\left(T+T_{\mathrm{s}}\right),
\end{aligned}
$$

where $T_{\mathrm{s}}$ is much smaller than $T$. Their phase difference vectors $Y_{0}, Y_{\mathrm{e}}$ are given by,

$$
\begin{aligned}
& Y_{0}=B \exp (j \Delta \theta)=B \exp \left(j \omega_{\mathrm{d}} T\right) \\
& Y_{\mathrm{e}}=B^{\prime} \exp \left(j \Delta \theta^{\prime}\right)=B^{\prime} \exp \left\{j \omega_{\mathrm{d}}\left(T+T_{\mathrm{s}}\right)\right\} .
\end{aligned}
$$

The vectors fluctuate due to noise. We therefore need to average these phase difference vectors according to the reflection waves obtained sequentially, that is

$$
\begin{aligned}
& X_{0}=\sum_{n=1}^{N} Y_{0}=D \exp \left(\overline{j \omega_{\mathrm{d}}} T\right) \\
& X_{\mathrm{e}}=\sum_{n=1}^{N} Y_{\mathrm{e}}=D^{\prime} \exp \left\{j \overline{\omega_{\mathrm{d}}}\left(T+T_{\mathrm{s}}\right)\right\} .
\end{aligned}
$$


In 2-D Doppler measurement, in order not to decrease the signal-to-noise ratio, the number of summations can be $N=4 \sim 8$ in each packet if a decreased frame rate is acceptable.

To calculate the second-order phase difference from pair of phase differences, $\Delta \Delta \theta\left(=\Delta \theta^{\prime}-\Delta \theta\right)$ (Fig. 3b), we first calculate $U$, the difference vector between two phase differences, from the complex multiplication between $X_{\mathrm{e}}$ and the complex conjugate of $X_{0}$. This is different from the complex division $X_{\mathrm{e}} / X_{0}$ used by Zrnić. ${ }^{16)}$

$$
U=X_{\mathrm{e}} \cdot \operatorname{conjg}\left[X_{0}\right],
$$

where 'conjg [ ]' means the conjugate of a complex number.

Substituting Eq. (5) into (6), we get

$$
\begin{aligned}
U & =D D^{\prime} \exp \left\{j\left(\Delta \theta^{\prime}-\Delta \theta\right)\right\} \\
& =D D^{\prime} \exp \left(j \omega_{\mathrm{d}} T_{\mathrm{s}}\right) \\
& =U_{\mathrm{r}}+j U_{\mathrm{i}}
\end{aligned}
$$

(where $U_{\mathrm{r}}$ : real part, $U_{\mathrm{i}}$ : imaginary part).

Consequently, Eq. (7) gives the difference between two phase differences (velocity) as follows.

$$
\begin{aligned}
\Delta \Delta \theta & =\Delta \theta^{\prime}-\Delta \theta \\
& =\omega_{\mathrm{d}} T_{\mathrm{s}} \\
& =\arg (U)=\operatorname{Arctan}\left(U_{\mathrm{i}} / U_{\mathrm{r}}\right),
\end{aligned}
$$

where we assume that the phase changes linearly and the acceleration is negligible in the each packet. Then Eq. (8) gives the Doppler angular frequency

From relationship (9), we get

$$
\omega_{\mathrm{d}}=\left(1 / T_{\mathrm{s}}\right) \cdot \operatorname{Arctan}\left(U_{\mathrm{i}} / U_{\mathrm{r}}\right) .
$$

$$
\left|\omega_{\mathrm{a}}\right| \leq \pi / T_{\mathrm{s}} \text { or }\left|f_{\mathrm{d}}\right| \leq 1 /\left(2 T_{\mathrm{s}}\right)
$$

(where " $f_{\mathrm{d}}$ " is the Doppler frequency).

In this proposed method, the maximum measurable Doppler frequency (velocity) of $1 /\left(2 T_{\mathrm{s}}\right)$ is reached when $\Delta \Delta \theta$ is $\pi$ (Fig. 3c). This is the main difference from the conventional method which can only measure up to $1 / 2 T$. New limitation is extended by $T / T_{\mathrm{s}}$ from the previous limitation of $\pi / T$.

Therefore, by selecting $T_{\mathrm{s}}$ to be shorter than $T$, for example, $T_{\mathrm{s}}=T / P(P>1)$, the measurable maximum velocity is extended $P$ times over the conventional value. For example, if we select $T=250 \mu$ s and $T_{\mathrm{s}}=T / P=250 / 5=50 \mu \mathrm{s}$, the maximum velocity is extended by $\left(P=T / T_{\mathrm{s}}\right) 5$ times over the conventional value. Thus, even high-speed blood flow can be measured without aliasing; it cannot be correctly measured with the conventional method (Fig. 3c).
2.3 Frequency Analysis using Non-equally-spaced Pulse Transmission ${ }^{15}$ )

We can also apply our proposed method to Doppler spectrum analysis. At each packet of several non-equally pulse transmission periods $T$ and $T+T_{\mathrm{s}}$, we can derive the averaged difference vector of two phase differences $U_{\mathrm{i}}$ as $\Delta \Delta \theta$ in Eq. (7). The direct products of second-order phase-difference vectors $U_{\mathrm{i}}$ are used to generate original phase vectors $S_{n}$.

$$
S_{n}=\prod_{k=1}^{n} U_{k}
$$

The Doppler frequency distribution can be obtained by taking the Fourier transformation of the regenerated phase vectors $S_{1} \sim S_{n} \sim S_{N}$, for example, $N=$ 128,256 . In order to prevent overflow or underflow in calculating the direct product of $S_{n}$ in Eq. (11), we need to normalize each $U_{k}$ by it's amplitude and furthermore multiply the product by the estimated amplitude value of the phase difference vector, for example, $\sqrt{\left|U_{n}\right|}$. Thus, as an alternative form of Eq. (11), we obtain

$$
S_{n}=\sqrt{\left|U_{n}\right|} \prod_{k=1}^{n} U_{k} /\left|U_{k}\right|
$$

The FFT (Fast Fourier Transformation) is performed on these regenerated phase vectors $S_{1} \sim S_{N}$ in Eq. (12) in order to produce a Doppler spectrum distribution. Thus, the measurable frequency domain is extended to $P\left(=T / T_{\mathrm{s}}\right)$ times that of the conventional method so that we get the de-aliased Doppler spectrum. This is because the phase shift during each sampling period is compressed $P$ $\left(=T / T_{\mathrm{s}}\right)$ times, that is, to within $\pm \pi\left( \pm 1 / 2 T_{\mathrm{s}}\right)$.

\section{COMPUTER SIMULATION}

In the experiment by computer software based on PAD (Program Analysis Diagram) (Fig. 4), a complex phase signal is firstly synthesized by computer with no random noise. Two kinds of phase difference vectors are each summed four times. Finally, we estimate the mean Doppler frequency by obtaining the argument of the complex multiplication value between $X_{\mathrm{e}}$ and the complex conjugate of $X_{0}$.

Table 1 shows results of the computer simulation. The left column is the input frequency, and the right columns are the estimated frequencies for $T_{\mathrm{s}}=60 \mu \mathrm{s}$, $10 \mu \mathrm{s}$. From Eq. (10), the theoretical maximum Doppler frequency for $T_{\mathrm{s}}=60 \mu \mathrm{s}$ is $8,333.3 \mathrm{~Hz}$, because the new maximum is $1 /\left(2 T_{\mathrm{s}}\right)$. The estimated Doppler frequency is precise within the range 


\section{H. NISHIYAMA and K. KATAKURA: NON-EQUALLY-SPACED PULSE TRANSMISSION}

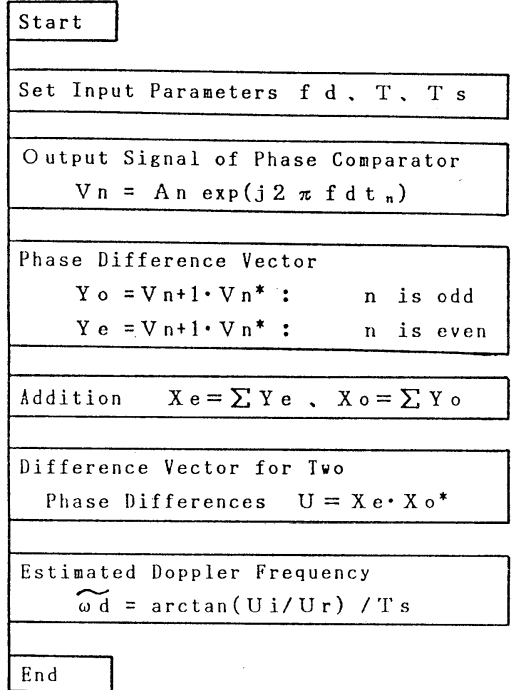

Fig. 4 PAD for computer simulation of proposed method (PAD: Program Analysis Diagram).

Table 1 Results of computer simulation of proposed method (constant velocity without noise).

\begin{tabular}{ccr}
\hline \multirow{2}{*}{$\begin{array}{c}\text { Input Doppler } \\
\text { frequency } \\
f_{\mathrm{d}}(\mathrm{Hz})\end{array}$} & \multicolumn{2}{c}{ Estimated Doppler frequency } \\
\cline { 2 - 3 } & \multicolumn{2}{c}{$\tilde{f}_{\mathrm{d}}(\mathrm{Hz})$} \\
\hline 0 & $T_{\mathrm{s}}=60 \mu \mathrm{s}$ & $T_{\mathrm{s}}=10 \mu \mathrm{s}$ \\
10 & 9.0 & 0.0 \\
100 & 99.99 & 9.99 \\
1,000 & 999.97 & 99.99 \\
5,000 & $5,000.64$ & 999.97 \\
6,000 & $6,000.14$ & $5,000.64$ \\
7,000 & $6,999.63$ & $6,000.14$ \\
8,000 & $8,000.71$ & $6,999.63$ \\
9,000 & $\times 7,666.49$ & $8,000.71$ \\
10,000 & $\times 6,667.0$ & $9,000.21$ \\
18,000 & $\times 1,333.2$ & $9,999.70$ \\
40,000 & - & $18,000.42$ \\
50,000 & - & $39,995.48$ \\
60,000 & - & $50,000.63$ \\
\hline
\end{tabular}

〈Theoretical value

for $T_{\mathrm{s}}=60 \mu \mathrm{s}, \quad(T=270 \mu \mathrm{s}), \quad\left|f_{\mathrm{d}}\right| \leqq 8,333.3 \mathrm{~Hz}$, for $T_{\mathrm{s}}=10 \mu \mathrm{s},(T=295 \mu \mathrm{s}),\left|f_{\mathrm{d}}\right| \leqq 50,000.0 \mathrm{~Hz}$.

predicted by the new maximum. With the conventional method, the maximum measurable Doppler frequency for $T=270 \mu$ s is about $1.8 \mathrm{kHz}$, which is $T_{\mathrm{s}} / T=60 / 270=1 / 4.5$ times lower than the maximum in our method. The theoretical value of maximum Doppler frequency for $T_{\mathrm{s}}=10 \mu \mathrm{s}$ is 50 $\mathrm{kHz}$, which is $T / T_{\mathrm{s}}=295 / 10=29.5$ times greater than with the conventional method. The estimated Doppler frequency is also precise up to the new maximum of $1 /\left(2 T_{\mathrm{s}}\right)=50 \mathrm{kHz}$. With the conventional method for $295 \mu \mathrm{s}$, the maximum frequency is $1.6 \mathrm{kHz}$.

The results by computer simulation for a single Doppler frequency with a high signal to noise ratio condition are in good agreement with the new method.

\section{EXPERIMENTAL}

We extract the Doppler signal from the output of a phase comparator in the Doppler unit of an ultrasonic diagnostic scanner EUB-40 made by HITACHI MEDICO. The transmitting circuit of EUB-40 was modified for non-equally-spaced pulse transmission intervals $T$ and $T+T_{\mathrm{s}}$. We store about 1 second of data digitalized with 8-bit resolution on Digital Memory DM-901 made by IWATSU, and transfer to a PDP11/34 mini-computer's disk, as shown in Fig. 5. Ultrasonic pulses are transmitted to a sample point which is at a fixed depth in the body. Using computer software to calculate Doppler velocity, velocity obtained by the proposed method and that obtained by the conventional method are printed out for comparison. We take the average over $4 \sim 8$ phase difference vectors to reduce the fluctuations in velocity due to noise. Furthermore, by taking the moving average of the difference vector of the phase differences $(4 \sim 16)$, we get the change in Doppler velocity against time.

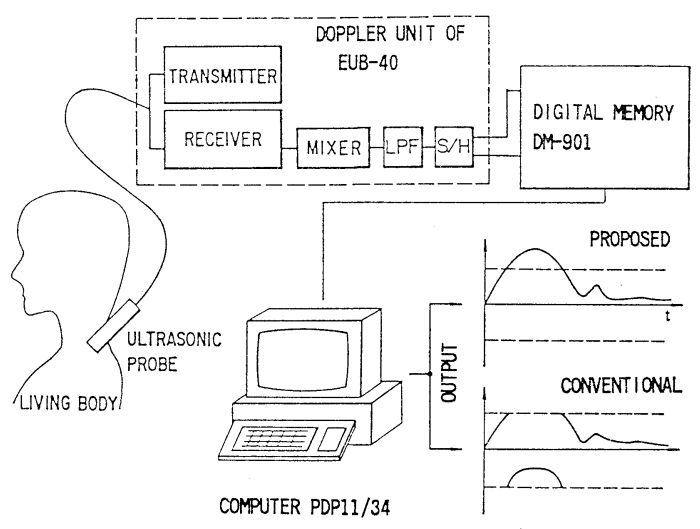

Fig. 5 Experimental setup. 


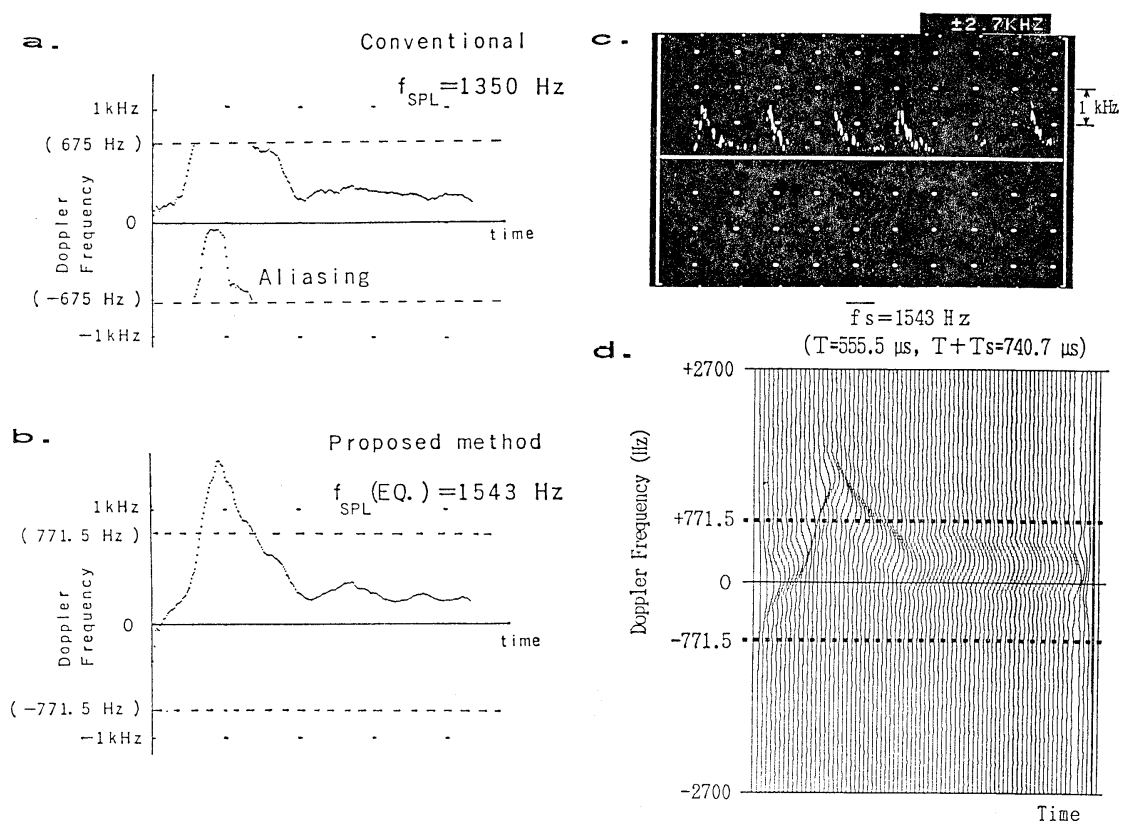

Fig. 6 Carotid artery blood flow: a. Conventional method. PRT $=740.8 \mu$ s. $\quad$ b. Proposed method. $P=T / T_{\mathrm{s}}=555.6 / 185.2=3$. Sum over 4 phase difference vectors, Moving average over 16 2nd-phase difference vectors. c. Pulsed Doppler equipment, EUB-40 Doppler ultrasound scanner is used by $P R F=5.4 \mathrm{kHz}$. d. Proposed frequency analysis. The 2ndphase difference vectors are obtained by the same condition as $b$.

In the frequency analysis, the FFT (Fast Fourier Transformation) used 128 data points consisting of 8 regenerated phase vectors and 120 added zero vectors point per processing frame, to get a Doppler frequency distribution. Furthermore, the each processing frame is formed from $2 / 3$ of the previous frame plus $1 / 3$ of the current frame in order to get the time-varying Doppler frequency distribution.

\section{EXPERIMENTAL RESULTS AND DISCUSSION}

\subsection{Carotid Artery Blood Flow Measurement}

We applied this method to clinical data on blood flow in the carotid artery. The horizontal axis in Fig. 6 shows the time corresponding to about a single cardiac cycle. In the conventional method, the Pulse Repetition Frequency PRF ( $=1 / \mathrm{PRT}=$ $1 / T=1 / 740.8 \mu \mathrm{s})$ is $1,350 \mathrm{~Hz}$. The Nyquist Frequency is then one half of PRF, that is, $675 \mathrm{~Hz}$. The alising phenomenon is clear with the conventional method (Fig. 6a). With the proposed method (Fig. 6b), however, there is no aliasing, and the mean two-pulse repetition frequency is $1 /\left\{\left(T+T+T_{\mathrm{s}}\right) / 2\right\}$
$=2 /\left(2 T+T_{\mathrm{s}}\right)=2 /(2 \times 555.6+185.2 \mu \mathrm{s})=1,543 \mathrm{~Hz}$, so the Nyquist frequency is $771.5 \mathrm{~Hz}$. With this method, we could measure the blood speed up to a new frequency limit of $2.7 \mathrm{kHz}$ without aliasing.

Figure $6 \mathrm{c}$ shows a result from the same area but taken by conventional Doppler equipment with a higher PRF of $5.4 \mathrm{kHz}$. The peak Doppler frequency is at about $1.5 \mathrm{kHz}$ but is almost the same as that obtained by the proposed method with a much lower PRF of $1,543 \mathrm{~Hz}$.

In the frequency analysis, the mean two-pulse repetition frequency is $1,543 \mathrm{~Hz}$. Figure $6 \mathrm{~d}$ shows the results of the proposed analysis. The movement of the peak frequency with time in the smoothed frequency distribution is similar to that taken by conventional Doppler equipment with a higher PRF of $5.4 \mathrm{kHz}$.

\subsection{Cardiac Blood Flow Measurement}

Figure 7 shows the measured cardiac blood flow with a low signal-to-noise ratio. In the conventional method (Fig. 7a), aliasing occurs above the Nyquist frequency of $333.5 \mathrm{~Hz}$, which is one half of 


\section{H. NISHIYAMA and K. KATAKURA: NON-EQUALLY-SPACED PULSE TRANSMISSION}

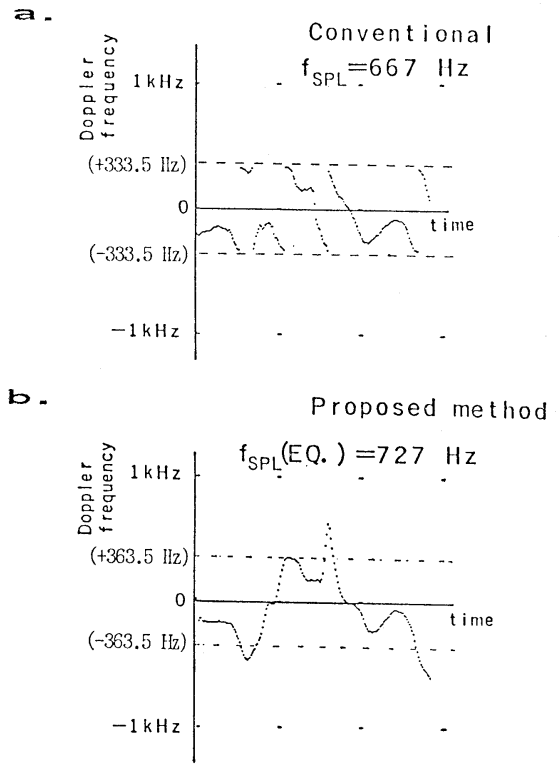

c.

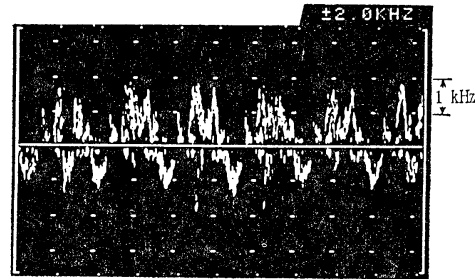

Fig. 7 Cardiac blood flow: a. Conventional method. PRT $=1,500 \mu \mathrm{s}$. b. Proposed method. $P=T / T_{\mathrm{s}}=1,250 / 250=5$. Sum over 4 phase difference vectors, Moving average over 12 2nd-phase difference vectors. c. Pulsed Doppler equipment, EUB-40 Doppler ultrasound scanner is used by $\mathrm{PRF}=4 \mathrm{kHz}$.

the PRF of $667 \mathrm{~Hz}$. In the proposed method (Fig. $7 b)$, where the mean two-pulse repetition frequency is $2 /\left(2 T+T_{\mathrm{s}}\right)=2 /(2 \times 1,250+250 \mu \mathrm{s})=727 \mathrm{~Hz}$, we can measure beyond the limit of the conventional method, up to a new maximum of $2 \mathrm{kHz}$. Figure $7 \mathrm{c}$ shows the result from the same area taken by conventional Doppler equipment with a higher PRF of $4 \mathrm{kHz}$. The change in Doppler frequency is found to be almost the same as that measured by the proposed method with a lower PRF of about $727 \mathrm{~Hz}$.

This figure shows that the frequency changes on both directions from the zero frequency axis (i.e., plus and minus), which differs from the results ob- tained by the zero-shift technique in the conventional method. With the zero-shift technique, the measured value may be twice as high on one side as on the other.

\section{CONCLUSIONS}

By computer simulation with a high signal-tonoise ratio, it is possible to measure a velocity 30 times that implied by the Nyquist frequency limit. We confirmed that we correctly measured not only the mean Doppler frequency (velocity) but also the spectral distribution of actual clinical data for carotid artery blood flow at frequencies over the Nyquist frequency. We also measured the mean Doppler frequency of cardiac blood flow which changes on both direction at over the Nyquist frequency. We showed that the new maximum measurable Doppler frequency is the inverse of twice the difference between the two different pulse repetition time ( $T$ and $\left.T+T_{\mathrm{s}}\right), 1 /\left(2 T_{\mathrm{s}}\right)$. Nonequally-spaced Pulse Transmission Method provides not only the mean Doppler frequency (velocity) but also the Doppler frequency distribution. However, further research is needed before this method can be applied to actual clinical situations: the signal-tonoise ratio must be increased.

\section{REFERENCES}

1) W. D. Rummler, "Introduction of a new estimator for velocity spectral parameters," Tech. Memo. MM-68-4121-5, Bell Telephone Lab. (1968).

2) K. Namekawa, C. Kasai, M. Tsukamoto, and A. Koyano, "Real-time blood flow imaging system utilizing autocorrelation technique," Proc. 3rd Conf. WFUMB, R. A. Lerski and P. Morley, Eds. (Pergamon, New York, 1982), pp. 203-208; K. Namekawa, A. Harada, and C. Kasai, "Realtime blood flow imaging system using Doppler ultrasound," IEICE J70-D, 1432-1440 (1987) (in Japanese).

3) C. Kasai, K. Namekawa, A. Koyano, and R. Omoto, "Real-time two-dimensional blood flow imaging using an auto-correlation technique," IEEE Trans. Sonics Ultrason. SU-32, 458-464 (1985).

4) V. L. Newhouse, P. LeCong, E. S. Furgason, and C. T. Ho, "On increasing the range of pulsed Doppler systems for blood flow measurement," Ultrasound Med. Biol. UMB-6, 233-277 (1980).

5) C. J. Hartley, "Resolution of frequency aliases in ultrasonic pulsed Doppler velocimeters," IEEE Trans. Sonics Ultrason. SU-28, 69-74 (1982).

6) R.S. Jaffe, "Extended-range pulsed Doppler meanfrequency estimation based on mean-frequency 
prediction," Tech. Rep. G556-3, Ph. D. Diss., Stanford Electronics Lab., Stanford Univ., Stanford, CA (1983. 5).

7) O. Bonnefous and P. Pesqué, "Time domain formulation of pulse-Doppler ultrasound and blood velocity estimation by cross correlation," Ultrason. Imaging 8, 73-85 (1986).

8) Y. Miyajima, K. Takamizawa, Y. Seo, and Y. Sumino, "Velocity measurement based on the time domain echo cross-correlation method," JSUM Proc. 54-118, 363-364 (1989) (in Japanese).

9) P. Tortoli, "A tracking FFT processor for pulsed Doppler analysis beyond the Nyquist limit," IEEE Trans. Biomed. Eng. BME-36, 232-237 (1989).

10) P. Tortoli, F. Valgimigli, G. Guidi, and P. Pignoli, "Clinical evaluation of a new anti-aliasing technique for ultrasound pulsed Doppler analysis," Ultrasound Med. Biol. 15 (8), 749-756 (1989).

11) K. R. Baek, M. H. Bae, and S. B. Park, "A new aliasing extension method for ultrasonic 2-dimensional pulsed Doppler systems," Ultrason. Imaging 11, 233-244 (1989).

12) K. Katakura, H. Nishiyama, and T. Ogawa, "Preliminary study for non-aliasing ultrasonic pulsed Doppler measurement," 32nd AIUM Proc., 126, New Orleans (1987. 10).

13) H. Nishiyama, T. Ogawa, and K. Katakura, "A new method of ultrasonic velocity measurement for very high-speed blood flow," IEICE Tech. Rep. US87-51, 5-9 (1987) (in Japanese).

14) H. Nishiyama, T. Ogawa, and K. Katakura, "Nonaliasing ultrasonic pulsed Doppler measurement," JSUM Proc. 52-144, 289-290 (1988) (in Japanese).

15) H. Nishiyama, T. Ogawa, and K. Katakura, "Nonaliasing ultrasonic Doppler frequency analysis," JSUM Proc. 54-122, 371-372 (1989) (in Japanese).

16) D. S. Zrnić and P. Mahapatra, "Two methods of ambiguity resolution in pulse Doppler weather radars," IEEE Trans. Aerosp. Electron. Syst. AES-
21, 470-483 (1985).

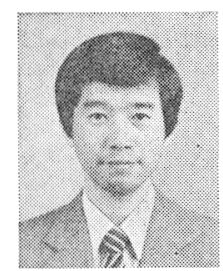

Hisashi Nishiyama was born in Kyoto, Japan, on March 1, 1951. He received the B.S. and M.S. degrees in mechanical engineering from Kyoto Institute of Technology, Kyoto, Japan in 1973 and 1975, respectively. In 1975, he joined the Central Research Laboratory, Hitachi, Ltd., Tokyo, Japan. He has been a researcher since 1981 and has been active in presenting papers at not only national meetings of the Japan Society of Ultrasonics in Medicine (JSUM), but also international symposiums on ultrasonic imaging and tissue characterization. His primary research interests are underwater signal processing, speech and medical applications, recently for color flow mapping. He is a member of the Japan Society of Ultrasonics in Medicine, the Acoustical Society of Japan and the Japan Society for Fuzzy Theory and Systems.

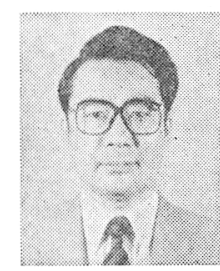

Kageyoshi Katakura was born in Saghalien, Japan, on March 26, 1944. He received the B.S., M.S. and Ph. D. degrees in electrical engineering from Tokyo Institute of Technology, Tokyo, Japan, in 1967, 1969 and 1988, respectively. In 1969, he joined the Central Research Laboratory, Hitachi, Ltd., where he was a Senior Researcher from 1978 to 1988. Since 1989, he has been a Chief Researcher at the Medical Electronics Research Dept. Dr. Katakura has been working to develop acoustic equipment for underwater and medical applications. $\mathrm{He}$ is a member of the IEICE of Japan, the Japan Society of Ultrasonics in Medicine and the Japan Society of Medical Electronics and Biological Engineering. He received the Satoh Paper Award in 1976. 